\title{
The Pakistan (Lahore) cochlear implant programme: issues relating to implantation in a developing country
}

\author{
M I J Khan, N MukHTAR*, S R SAEed $\dagger$, R T RAMSDEn†
}

\begin{abstract}
The Pakistan cochlear implant programme was started in Lahore in August 2000. It was initially established with the technical support of the Manchester cochlear implant team. There are no government resources available for cochlear implantation in Pakistan and the cost of cochlear implantation is met by the candidates and their families. Up till December 2005, 52 individuals have been implanted. Forty-four (84.6 per cent) of these patients were children and eight (15.4 per cent) patients were adults. Congenital deafness ( 94 per cent) is the main aetiological factor in children. Seventy-six per cent of these children were born to blood related parents. All patients received a Med-El Combi $40+$ device. The listening progress profile, the meaningful auditory integration scale and the meaningful use of speech scale were used to assess the auditory performance in children. Thirty-nine children achieved an average listening progress profile score of 37.7 after 12 months of implantation. The overall rate of major and minor complications was 11.5 per cent. Failure rate for the device itself has been 3.8 per cent. In addition to considering these preliminary outcomes and funding issues, the problems of setting up a cochlear implant programme in a developing country are discussed.
\end{abstract}

Key words: Cochlear Implants; Sensorineural Deafness; Developing Countries

\section{Introduction}

Cochlear implantation has become an established means of auditory rehabilitation in selected severely and profoundly deaf adults and children. ${ }^{1,2}$ Its efficacy, safety and reliability are well recognised. Over 100000 patients have received cochlear implants worldwide and children are emerging as the largest group of patients to benefit. Technology is evolving and the candidacy criteria are widening. Bilateral cochlear implantations in children and combined electroacoustic stimulation are the new developments in this field of rehabilitation for the deaf. ${ }^{3}$

According to the World Health Organization (WHO) more than 80 per cent of the world's 120 million people who have disabling hearing difficulties live in developing countries. ${ }^{4}$ Only a few countries like China, Saudi Arabia, Iran, Brazil, Egypt and Malaysia have been able to establish reliable cochlear implant programmes. However, these are the relatively richer countries in the developing world and have good public sector health provision systems. There are financial implications for the delivery of cochlear implant programmes; and when taken into consideration with other factors, it makes the candidacy criteria very different from those in the developed world. ${ }^{5}$ Pakistan is a country with a population of 150 million. Using the WHO figure of 10 per cent it has 15 million people with hearing disorders. We estimate that there are 150000 congenitally deaf individuals. This implies that Pakistan has a large population of deaf individuals who could potentially derive benefit from cochlear implantation.

In this paper we describe our experience of developing a cochlear implant programme in Pakistan and the trends and initial outcomes of the programme.

\section{Material and methods}

\section{Setting up the programme}

The Pakistan cochlear implant programme started in 2000. Prior to the development of this programme patients travelled to Europe and North America for cochlear implantation. Due to financial implications very few patients could afford this and there were difficulties with rehabilitation and long-term follow up.

The Pakistan cochlear implant programme started in the private sector. We endeavoured to seek government funding from the outset but financial constraints made this task impossible. A core team

From the Department of Otolaryngology \& Head and Neck Surgery, Bradford Teaching Hospitals NHS Foundation Trust, Bradford, UK (at the Sir Ganga Ram Hospital Lahore, Pakistan from 1999-2002), the *Audiology Centre, Lahore, Pakistan, and the $†$ Department of Otolaryngology, Manchester Royal Infirmary, Manchester, UK.

Accepted for publication: 28 January 2007. 
comprising an ENT surgeon, audiological physician, speech and language therapist, teacher for the deaf and clinical psychologist were assembled. It was initiated with the technical support and advice of the cochlear implant team at the University of Manchester. The programme aims to provide services to both adults and children. There were no minimum or maximum age limits for referral and assessment.

After clinical assessment and audiological evaluation, the appropriateness of the candidates was assessed. Children at or above the age of seven years, and who were congenitally deaf, and had not used hearing aids, did not receive cochlear implantations. Those individuals or families who could afford the cost of the device, surgery and subsequent rehabilitation and had a commitment to the rehabilitation programme were counselled with a view to proceeding to implantation. In addition, only those individuals or families who had access to a speech therapist and to a teacher for the deaf locally or who could move to Lahore for rehabilitation were offered the implant.

\section{Patients}

Data relating to the patients who received cochlear implantation between August 2000 and December 2005 were analysed. A total of 54 implant operations were performed on 52 patients. The patients were divided into paediatric and adult groups. The paediatric group was subdivided into those receiving cochlear implants before the age of five years and at or over the age of five years. The age at implantation, aetiology of deafness and its duration and other associated conditions were determined. History of consanguinity was also recorded. Place of domicile was also determined because it was a major factor with regard to commitment to the rehabilitation programme. All patients received a multichannel cochlear implant (Combi 40+, MED-EL medical electronics, Innsbruck, Austria).

Post-operative switch on and fitting was performed four weeks after the surgery. Each patient received individual auditory and communication skills training and their progress was assessed using listening progress profile scoring in children and monosyllabic word score and the Glendonald auditory screening procedure in adults. The auditory skills and communication strategies of the children in the over the age of five years group were assessed by using the meaningful auditory integration scale and the meaningful use of speech scale. The patients were rehabilitated in English as well as in Punjabi and Urdu, depending upon their mother tongue. Most children and all adults were rehabilitated in at least two languages.

Both audiological and surgical complications were recorded. The overall complications rate and incidence of major and minor complications were measured.

\section{Results}

A total of 54 implantations have been performed on 52 patients (Figure 1). All patients received Med-El

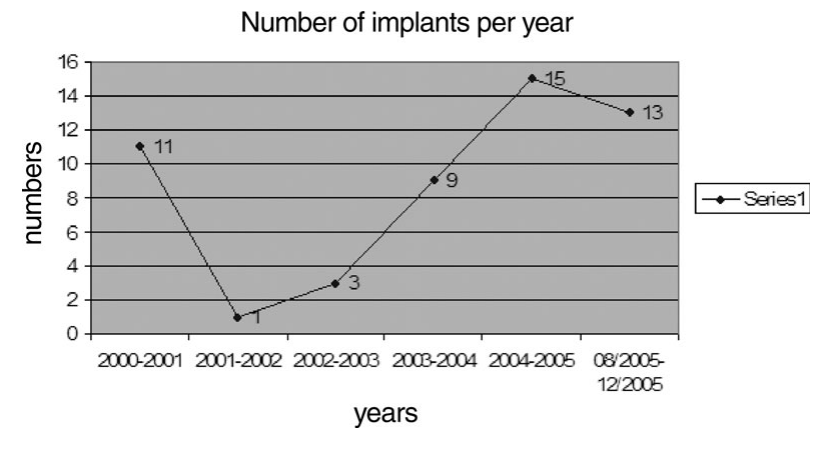

Fig. 1

Number of implants per year.

Combi40+ devices. Forty-four (84.6 per cent) of these patients were children (male, 23; female, 21) (Figure 2). Eight (15.4 per cent) patients were adults (male, five; female, three). Twenty-six (59 per cent) children received cochlear implants before five years of age. The average age of the patients in this subgroup was 34 months (range 16-52 months; standard deviation [SD] 10.95). The median age was 33 months. All of these children were congenitally deaf. Eighteen (41 per cent) children had their cochlear implant surgery at or above the age of five years. The average age in this group was 85.3 months (range 61-173 months; SD 26.97). The median age was 78 months. In the paediatric group 94 per cent had congenital deafness (Table I). Other aetiologies included mumps 2 per cent, kernicterus 2 per cent and meningitis 2 per cent.

Seventy-six per cent of these children were born to blood related parents. None of the children had deaf parents. One patient had deaf siblings who did not have cochlear implants. One patient had prune belly syndrome (abdominal wall defect, genitourinary anomalies and musculoskeletal anomalies). Leukodystrophy of the brain was discovered in one patient on magnetic resonance imaging (MRI) prior to surgery. None of the patients had any inner-ear anomalies on MRI/computed tomography imaging.

In the adult group, the average age at cochlear implantation was 21.93 years (range 16.08-29.16 years). Three patients (37.5 per cent) had congenital deafness (Table II). Other aetiologies included progressive sensorineural hearing loss in two patients

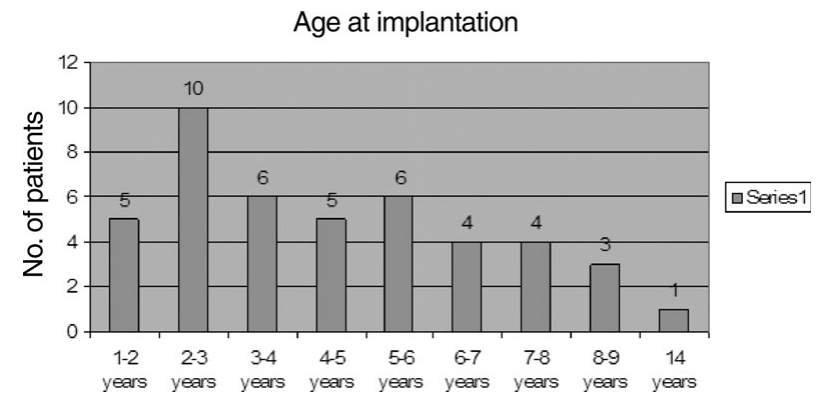

Fig. 2

Age at implantation in paediatric group. 
TABLE I

AETIOLOGY OF DEAFNESS IN PAEDIATRIC GROUP

\begin{tabular}{lc}
\hline Aetiology & No. (\%) \\
\hline Congenital & $41(94)$ \\
Meningitis & $1(2)$ \\
Kernicterus & $1(2)$ \\
Mumps & $1(2)$ \\
\hline
\end{tabular}

(25 per cent), meningitis two patients ( 25 per cent) and Ehlers-Danlos syndrome in one patient $(12.5$ per cent).

The overall incidence of complications was 11.5 per cent. There were minor complications in 5.77 per cent that included transient wound swelling in one patient, and late onset localised inflammation on the scar area in another patient (Table III). Subsequent exploration of the area revealed a small piece of bone wax in the subcutaneous tissue. There were no major surgical complications. One patient had recurrent hyperaemia of the scar area which was rectified by changing the magnet strength. One patient suffered from non-auditory stimulation in the form of pain and discomfort. There were two (3.84 per cent) device failures in a total of 52 primary implant surgeries. These were successfully re-implanted. These devices failed about 26 months after the primary surgery. Both of these patients had primary implant surgery on consecutive days. The manufacturers explained that these two devices came from a batch of devices with an inherent fault. This has since been rectified.

To date there have been no non-users of the device. The auditory performance of the children was assessed by the listening progress profile. Thirtynine children have more than 12 months' use of cochlear implants and they had an average listening progress profile score of 37.56 after 12 months of cochlear implantation (Figure 3). The meaningful understanding of speech scale and the meaningful auditory integration scale scores were obtained in 17 children in the older than five years age group (Figure 4). These patients have an average follow up of 30.9 months (range $66-6$ months). The mean pre-implant meaningful use of speech scale and meaningful auditory integration scale scores were 2.58 (range 0-20; SD 6.57) and 2.88 (0-22; SD 6.61), respectively. After a minimum six month's use of the implant, the average post-implant meaningful use of speech scale and meaningful auditory integration scale scores were 17.41 (range 2-39;

TABLE II

AETIOLGY OF DEAFNESS IN ADULT GROUP

\begin{tabular}{ll}
\hline Aetiology & No. (\%) \\
\hline Congenital & $3(37.5)$ \\
Progressive & $2(25)$ \\
Meningitis & $2(25)$ \\
Mixed & $1(12.5)$ \\
\hline
\end{tabular}

TABLE III

COMPLICATIONS

\begin{tabular}{ll}
\hline Complications & No. (\%) \\
\hline Wound complications & $3(5.77)$ \\
$\quad$ Transient wound swelling & $1(1.92)$ \\
Localised inflammation & $2(3.84)$ \\
Non-auditory stimulation & $1(1.92)$ \\
Device failure & $2(3.84)$ \\
\hline
\end{tabular}

SD 12.21) and 29.94 (range 16-40; SD 9.22). In one patient we could not get the scores as she had relocated abroad.

Five post-lingual adult recipients achieved an average open set monosyllabic word score of 96 per cent and all of them scored 100 per cent on the Glendonald auditory screening procedure. All of them are using the telephone successfully. One of these adult patients can converse in three languages and on most occasions could recognise the person on the phone. Two of the adults had congenital deafness which progressed during early childhood. They were using a hearing aid and had good speech development. Their open set monosyllabic word scores and Glendonald auditory screening procedure scores were 72 per cent, 70 per cent, 68 per cent and 50 per cent, respectively.

\section{Discussion}

The cost effectiveness of cochlear implantation in selected deaf individuals is very well established. ${ }^{1,2,15}$ However, cochlear implantation is a low volume, high cost medical intervention. In spite of tremendous improvements in the technology of cochlear implantation, the cost of the device has remained high (between $\$ 15000$ and $\$ 35000$, depending upon the manufacturers and local market). Consequently most of the cochlear implant recipients are in the developed countries of North America, Europe and Australia.

Since the 1980s, there have been some reports of the use of cochlear implants for the rehabilitation of deaf individuals in the developing countries; only a handful of countries such as China, Saudi Arabia, Iran, Egypt, Brazil and Malaysia have established cochlear implant programmes. ${ }^{4,5,6,7}$ These countries are relatively rich among the developing countries and could afford a cochlear implant programme funded by the government sector.

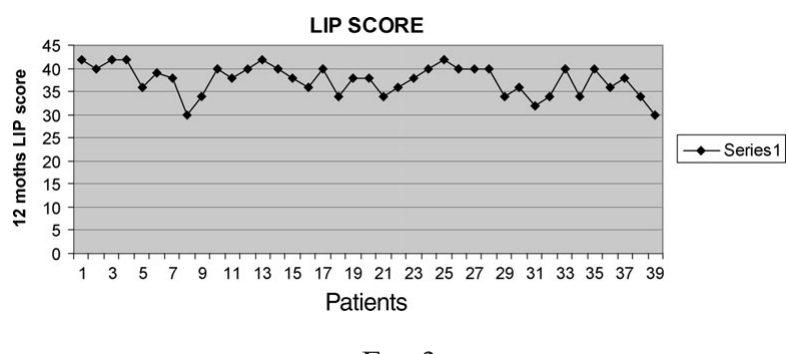

FIG. 3

12 month listening progress profile scores. LIP $=$ listening progress profile 
(a)

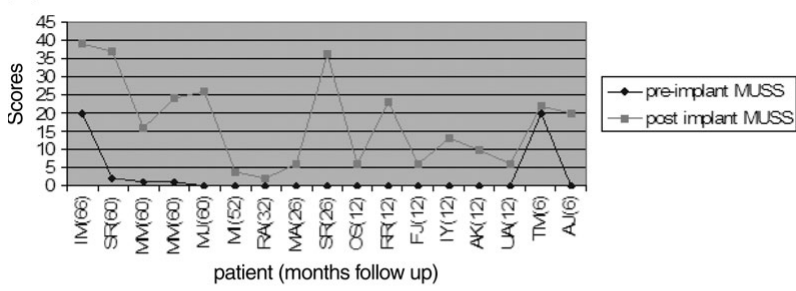

(b)

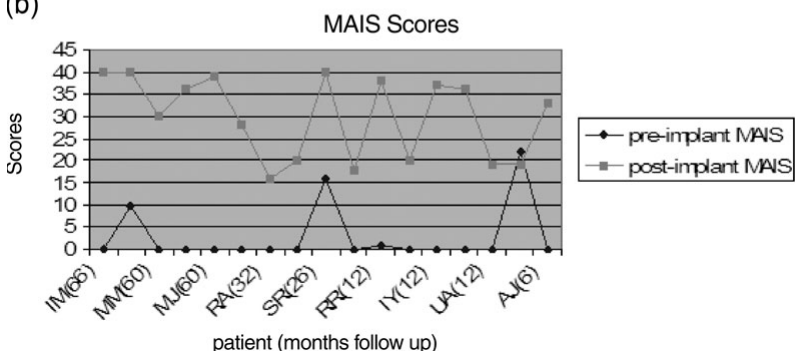

FIG. 4

(a) Meaningful use of speech scale and (b) meaningful auditory integration scale scores in children in the older than five years age group. MUSS = meaningful use of speech scale MAIS = meaningful auditory integration scale

Pakistan is one of the poorest countries in the developing world, with a per capita income of $\$ 600$. According to the 1981 census, 13 per cent of those with a disability were deaf individuals. ${ }^{8}$ There are no government funded centres which could provide rehabilitation services for the deaf individuals. Only very few tertiary centres have audiology departments and in most centres hearing aid devices are paid for by the patients. According to the WHO, an estimated 32 million hearing aids are needed every year in the developing countries: but less than one million are actually fitted with hearing aids. ${ }^{9}$ Based on these facts, we started our cochlear implant programme on a self-finance basis. This was the only way forward to overcome the financial hurdles.

Our experience of setting up a cochlear implant programme in Lahore on a self-finance basis is not unique in itself; India has similar programmes established in major cities (Dr Mohan, Dr Manoj and Med-EL Austria, personal communication). These programmes face quite peculiar problems with regards to the selection of patients, team of professionals and the device itself.

The patient demographics are quite different from those in the developed world. Of the cochlear implant recipients in our series, 84.6 per cent were children. Only eight adults (15.4 per cent) have had a cochlear implantation since the start of our programme in 2000. Considering all the factors including financial issues, one would have expected at least the same number of adults as children to have had cochlear implants. Mawman et al. reported similar trends in a Manchester adult cochlear implant programme in which the ratio of adult patients of Pakistani origin being implanted is disproportionately low to the numbers in the population as a whole. ${ }^{10}$ This is in contrast to the paediatric programme which has many more deaf children from Asian families who have received cochlear implantations.

We feel that this is a reflection of the attitude of society towards deafness as a disability. It reiterates the fact that most people in a society, such as that of Pakistan, do not pay much attention to deafness. In Pakistan, one could argue that financial constraints would put cochlear implantation for adults low in the scale of priorities. However, similar trends in British adults of Pakistani origin indicate that other cultural and social factors are also involved.

Among Asian families, consanguinity is a major factor in congenitally deaf children. There is one epidemiological study in Pakistan which revealed that in cases of severe hearing loss, 70 per cent were the result of consanguineous marriages. ${ }^{11}$ In our series, 76 per cent of the children with congenital deafness were born to blood related parents.

Selection criteria are much more stringent and, in some respects, different than in the developed countries. ${ }^{5}$ In our programme, at the very outset of pre-implant assessment stage it is determined whether a candidate has sufficient financial resources to afford the cost of implantation and subsequent rehabilitation. It means that many suitable candidates who could have benefited from cochlear implantation are declined due to lack of financial resources. Access to the rehabilitation is only available in two or three major cities in the country. The potential candidate should be able to attend the rehabilitation programme which means that only those individuals living in the major cities or having easy access to these cities could be considered for implantation. Seventy-six per cent of our cochlear implant recipients reside in Lahore and Karachi, which are the two largest cities in the country. It also has to be determined whether the potential candidate, or the family of a child, is well motivated and committed to the rehabilitation process. Their expectations should be realistic and it needs careful and thorough pre-implant counselling. We had an arrangement whereby potential candidates and their families met existing cochlear implant users, while they went about their routine day to day life. Our experience with such arrangements indicates that this provides a tremendous background for counselling and helps the potential candidates and their families to develop an understanding of cochlear implant usage and its benefits.

It is important that the potential candidate and the family of a candidate are well motivated and highly committed to the rehabilitation process. Other factors such as financial resources to bear the ongoing cost of the rehabilitation and maintenance of the device (batteries, repairs) are also considered. These factors are important to avoid device non-use. In our cohort of patients, the number of device non-use was zero.

In most of the developing countries the professionals working in the cochlear implant teams are either trained or qualified in the West. The Pakistan cochlear implant programme had an otolaryngologist and an audiological physician both 
trained at the University of Manchester. The programme was started with initial technical support from the Manchester cochlear implant team. Such arrangements of 'dovetailing' of the local professionals with members of an established programme are crucial to set up a cochlear implant programme of the highest standard and these help to earn the confidence of other local professionals.

Multichannel cochlear implants yield the best results and there is not much difference in the outcomes with different types of cochlear implants. ${ }^{10}$ We used Med-El Combi40+ devices in all our patients.

Cochlear implantation has been established as a safe intervention. However, as any other surgical intervention, it has an inherent risk of minor or major complications. The overall incidence of complications is reported as between $7-37$ per cent in the literature. ${ }^{12}$ In our series of patients the overall rate of complications related to surgery itself was 5.77 per cent. There was one patient who had transient wound swelling post-operatively. It resolved with conservative management. One patient developed localised inflammation in the scar area and exploration of the area revealed a small piece of bone wax causing localised inflammation. To date, we have had no major complications related to surgery. We have two device failures (3.84 per cent) which were re-implanted successfully.

There are reports of meningitis following cochlear implant surgery. The majority of these cases have been reported in the United States. ${ }^{13}$ To date we have had no cases of meningitis following cochlear implant surgery. We routinely vaccinate patients against Streptococcus pneumonae before implantation.

The listening progress profile was devised to monitor the development of auditory perception in the early stages after cochlear implantation. We used this test for two reasons; firstly the children have very limited communication skills and secondly their first language is not English. There are no standardised tests in Urdu or Punjabi (the two most commonly spoken languages in Pakistan) to assess speech and language development. It is a sensitive measure to assess the progress in speech development in very young children. Our mean listening progress profile scores at 12 months are in line with those reported in a series from Nottingham. ${ }^{14}$

- Cochlear implant services can be established in developing countries although cost remains the main limiting factor

- Children are the principal recipients of cochlear implants with consanguinity being the major factor in congenital deafness

- Very few of the suitable adult candidates chose cochlear implantation

- Technical support from established implant programmes and the implant companies is crucial for a successful programme
There is no doubt that cochlear implantation in children should be performed at a young age, preferably in infancy. Nonetheless, although we recommend congenitally deaf children receive cochlear implantation before the age of five years, there were 18 (41 per cent) children who were implanted between five and 14 years of age. This higher proportion of older children in our programme was mainly due to two factors. Firstly, as with any other cochlear implant programme in the world, we had a cohort of older children at the start of the programme. Secondly, the whole pathway of auditory impairment detection, pre-implantation assessment and arrangement of funds could take a long time and more often than not, children fell into the unfavourable group whilst still waiting for the funding. These are the peculiar difficulties a cochlear implant team faces in developing countries.

However, these older children received substantial benefit from cochlear implantation across multiple domains. The self-confidence and improved social interaction was greatly valued by the parents (S Anjum et al., unpublished data). In a society like Pakistan, hearing disability is a stigma. There are cultural issues and issues of parental sensitivities. There is no well-defined deaf culture and deaf individuals not only face the dilemma of deafness but of cultural identity as well. We recognise that the overall effect of cochlear implantation in these children is substantial.

\section{Conclusion}

Cochlear implant programmes can be successfully established in developing countries. Government funding is available in only a few countries and most countries are unable to fund such programmes from the government sector. Financial constraints are the major factor in keeping the number of cochlear implants to its current limited levels. To achieve the highest safety standards, support from an established implant programme is crucial. Cochlear implant companies have a role to play in terms of providing technical support to the professionals and prompt response and support to the patients in situations such as device failure. The use of cochlear implants by suitable candidates will remain limited in the foreseeable future and children should be the main group of cochlear implant recipients. Cochlear implant programmes play a role in raising awareness about deafness in society and have long lasting effects on cultural trends, for example issues related to consanguinity.

\section{References}

1 Summerfield AQ, Marshall DH. Cochlear implantation in the UK 1990-1994: Report by the MRC Institute of Hearing Research on the evaluation of the national cochlear implant programme. Her Majesty's Stationary Office, London, 1995

2 Cheng AK, Rubin RH, Powe NR, Mellon NK. Cost-utility analysis of the cochlear implant in children. JAMA 2000; 284:850-6 
3 Ramsden RT. Prognosis after cochlear implantation. $B M J$ 2004;328:419-20

4 Zeng FG. Cochlear implants in developing countries. Contact 1996;10:5-9

5 Berruecos P. Cochlear implants: An international perspective-Latin American countries and Spain. Audiology 2000; 39:221-6

6 Belal A. Cochlear implantation in developing countries. Am J Otol 1986;7:244-7

7 Farhadi M, Daneshi A, Emamjomeh H, Hasanzadeh S. Cochlear implantation in Iran: a report of 190 cases. $A d v$ Otorhinolaryngol 2000;57:435-8

8 Ahmed T. The population of persons with disabilities in Pakistan. Asia Pac Popul J 1995;10:39-62

9 Kumar S. WHO tackles hearing difficulties in developing world. Lancet 2001;358:219

10 Mawman DJ, Bhatt YM, Green KMJ, O'Driscoll MP, Saeed SR, Ramsden RT. Trends and outcomes in the Manchester adult cochlear implant series. Clin Otolaryngol 2004:29:331-9

11 Elahi MM, Elahi F, Elahi A, Elahi SB. Paediatric hearing loss in rural Pakistan. J Otolaryngol 1998;27:348-53

12 Green KMJ, Bhatt YM, Saeed SR, Ramsden RT. Complications following adult cochlear implantation: experience in Manchester. J Laryngol Otol 2004;118:417-20
13 Josefson D. Cochlear implants carry risk of meningitis, agencies warn. BMJ 2002;325:298

14 Nikolopoulos TP, Wells P, Archbold SM. Using Listening Progress Profile to assess early functional auditory performance in young implanted children. Deafness and Education International 2000;2:142-51

15 Cheng AK, Niparko JK. Cost-utility of the cochlear implant in adults: A meta-analysis. Arch Otolaryngol Head Neck Surgery 1999;125:1214-18

Address for correspondence:

M I J Khan,

Department of Otolaryngology,

Bradford Royal Infirmary, Duckworth Lane,

Bradford BD9 6RJ, UK.

Fax: 44 (0)1625533109

E-mail: mijkhan@yahoo.com

Mr M I J Khan takes responsibility for the integrity of the content of the paper.

Competing interests: None declared 